\title{
On the colour and spin of epistemic error (and what we might do about it)
}

\author{
K. Beven ${ }^{1,2,3}$, P. J. Smith ${ }^{1}$, and A. Wood ${ }^{1,4}$ \\ ${ }^{1}$ Lancaster Environment Centre, Lancaster University, Lancaster, UK \\ ${ }^{2}$ Department of Earth Sciences, Geocentrum, Uppsala University, Uppsala, 75236, Sweden \\ ${ }^{3}$ Centre for the Analysis of Time Series (CATS), London School of Economics, London, UK \\ ${ }^{4}$ JBA Consulting, Warrington, UK
}

Received: 5 May 2011 - Published in Hydrol. Earth Syst. Sci. Discuss.: 30 May 2011

Revised: 25 August 2011 - Accepted: 26 August 2011 - Published: 13 October 2011

\begin{abstract}
Disinformation as a result of epistemic error is an issue in hydrological modelling. In particular the way in which the colour in model residuals resulting from epistemic errors should be expected to be non-stationary means that it is difficult to justify the spin that the structure of residuals can be properly represented by statistical likelihood functions. To do so would be to greatly overestimate the information content in a set of calibration data and increase the possibility of both Type I and Type II errors. Some principles of trying to identify periods of disinformative data prior to evaluation of a model structure of interest, are discussed. An example demonstrates the effect on the estimated parameter values of a hydrological model.
\end{abstract}

\section{Introduction}

The starting point for this paper is the belief in certain parts of the modeling community that it is necessary to use a statistical framework to evaluate the uncertainty in model predictions. This has been the subject of much discussion in the past, with a range of positions from the pure (even if Bayesian) probabilistic views of, for example, O'Hagan and Oakley (2004), Goldstein and Rougier (2004), Mantovan and Todini (2006), Stedinger et al. (2008) and others, to the sceptical views of Beven (2006) and Andréassian et al. (2007). Statistical treatments of errors have been applied quite widely in hydrological modelling, developing from the use of likelihoods based on assumptions about model residuals (e.g. Sorooshian and Dracup, 1980) to the much more

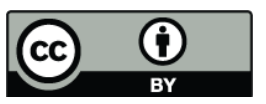

Correspondence to: $\mathrm{K}$. Beven (k.beven@lancs.ac.uk) sophisticated hierarchical treatments of multiple sources of error exemplified by the BATEA (Kuczera et al., 2006; Thyer et al., 2009; Renard et al., 2010) and DREAM (Vrugt et al., 2008, 2009) methodologies.

There are two main advantages of this approach. The first is that it provides a formal framework underpinned by decades of development of statistical methods, including for example the use of Monte Carlo Markov Chain techniques within a Bayesian framework for evaluating the posterior distributions when new data are added. The second is that it aims to provide an estimate of the probability of predicting an observation conditional on a particular model structure (or structures in Bayesian model averaging) and calibration data set (though note that Bayes original 1763 formulation was not in this form). We will not consider further cases where only forward uncertainty estimation can be carried out. Uncertainty estimates then depend entirely on the prior assumptions about different sources of uncertainty. Conditioning on some calibration data makes the problem much more interesting.

However (as noted by the referees), it is necessary to be very careful about terminology here. "Statistics" can apply to the analysis of any sample of numbers (originally referring to information about a State). As such it can apply to both formal and informal methods of manipulating data, although it is now normally reserved for methods that are founded in the axioms of probability. The questions we raise here about the application of formal statistical methods to series of hydrological model residuals are concerned with whether the assumptions required for a formal statistical analysis are valid or appropriate. These assumptions generally imply a rather simple stationary model of the residuals but a consideration of the way in which different types of uncertainty affect the

Published by Copernicus Publications on behalf of the European Geosciences Union. 
modelling process would suggest that the assumptions will be too simplistic.

However, the objectivity claimed for formal statistical methods, in this type of application, lies in the possibility of testing those assumptions against the summary statistics for a particular set of model residuals. It should be good practice in any modelling study to carry out such checks (though this is not often reported in papers based on formal statistical likelihoods). If those assumptions cannot be shown to be valid then, despite the mathematical formalism of the consequent inference, there is no objectivity. There may also be some limitations of this kind of objectivity when a number of different sets of assumptions appear to be acceptable, such that a subjective choice between different (formal) error models must be made. In Bayesian statistics there is also the subjective choice of prior distributions that, in some applications, have a significant effect on the resulting posterior distributions. It can also be shown (see for example Beven et al., 2008) that an objective analysis of one data set can lead to erroneous forecasts of new data. This paper discusses one reason why such a situation may arise; the role of epistemic uncertainties in determining the nature of a series of model residuals. It is suggested that the assumptions of formal statistics might be inappropriate in assessing the information content in such cases. Consequently this may result in misleading inference about model parameters and forecasts.

The main alternative to the formal statistical approach, at least up to now, has been the Generalised Likelihood Uncertainty Estimation (GLUE) methodology, first introduced by Beven and Binley (1992). GLUE is consistent with hierarchical Bayesian methods in that if an error model component is added and the associated likelihood based on formal statistical assumptions is used, then the results should be the same (Beven et al., 2007, 2008). However, GLUE also allows informal likelihoods (or fuzzy measures) to be used, can treat residual errors implicitly in making predictions, and provides ways of combining likelihoods other than Bayesian multiplication, where this seems appropriate. There are some conditions under which the resulting posterior likelihoods can be considered as probability distributions (see Smith et al., 2008), but the meaning will be different. When an informal likelihood is used, the resulting predictions will no longer formally be conditional estimates of the probability of predicting an observation, but rather conditional estimates of the probability of a model prediction. This has led to significant criticism of the GLUE method (e.g. Mantovan and Todini, 2006; Stedinger et al., 2008); though both of these critical studies are based on hypothetical examples where the model structure is known to be correct so that there is no epistemic uncertainty (see the extension of one of these cases to an incorrect model structure in Beven et al., 2008).

Experience of using the GLUE methodology with a variety of different likelihood measures suggests that for cases where the ensemble of model predictions can cover the available observations (e.g. in hypothetical examples with a correct model structure) the resulting estimates of model uncertainty can be rather similar. They will differ more in cases where a model structure is, for whatever reason, biased in part or parts of the calibration in the sense that it is impossible for that model structure to match an observation regardless of choice of parameter values. Some simple error structure, such as constant bias or a simple trend, can be easily handled in a statistical likelihood approach (e.g. Kennedy and O'Hagan, 2001) as can constant heteroscedastic variations in the error variance or a constant autocorrelation function. Time variable changes in bias or variance are more difficult (they suggests that the error series does not have a simple statistical structure) but can be "handled" in the sense of increasing the error variance of the identified error model, even in cases when the "best available" (maximum likelihood) model might not actually be fit for purpose, or where the source of the error comes from the poor specification of inputs for one or more events (though note that separating these cases might often be difficult).

In doing so, however, the validity of the model structure as a hypothesis about how the system is functioning will not be questioned. Since the statistical estimates of uncertainty are always conditional on the choice of model(s), there is no inherent testing of the validity of the model as hypothesis. Different models can be tested relative to each other (e.g. by the use of Bayes ratios) but there is no mechanism for total model rejection.

This is different from the GLUE approach which developed out of the earlier Hornberger-Spear-Young (HSY) generalized sensitivity analysis (Hornberger and Spear, 1981). The HSY method investigates the sensitivity of complex systems by differentiating between those models considered to be "behavioural" and those that can be rejected as "nonbehavioural". In that the predictions of model outputs within GLUE are intended to be useful guides to the future outputs from the system, there is no point in making predictions with models that have not proved to be behavioural in calibration. Thus models not thought to be useful in prediction are rejected (or given a likelihood of zero; in the statistical approach a very small likelihood would be given to such a model, but no model would be rejected).

However, this introduces a further degree of subjectivity in GLUE. There is commonly a complete range of behaviours in calibration between models that fit the data well (or as well as might be expected) and those that clearly do not. Thus, deciding on a threshold between what will be considered behavioural and what will not is necessarily subjective (even if generally common sense will prevail in doing so; see the limits of acceptability approach suggested in Beven, 2006). The effect of this selection can be mitigated to some extent by using an informal likelihood measure that reduces to zero at the rejection limit.

Such subjectivity is, of course, anathema to many scientists. For example, one referee highlights the difficulty of subjecting informal likelihoods to rigorous testing. The aim 
of science is to be as objective as possible, even if the history of science records very many instances of the subjective and selective use of evidence in many different subject areas: the history of using the Hortonian model to explain storm runoff is just such an example in hydrology (see Beven, 2004). We like to have formal frameworks for doing things, in which the consequences follow directly and straightforwardly from the assumptions. So why should we even consider using informal likelihoods and subjectively chosen thresholds? In fact, a somewhat deeper reflection turns that question around. How can we possibly justify the use of statistical error models and formal likelihoods when many of the errors that affect modeling uncertainty in hydrology are not "statistical" in nature?

\section{Aleatory and epistemic errors}

The application of formal statistical methods requires that the representation of errors is fundamentally as random or aleatory variables. Aleatory errors can be represented in terms of the odds (or probabilities) of different outcomes. The original paper of Bayes (1763), for example, was concerned with estimating odds on different potential hypotheses when there might be some prior (subjective) beliefs about the hypotheses. Bayes equation then provides a formal means for incorporating evidence as represented by a likelihood function with the subjective prior beliefs to condition a posterior distribution in a way that satisfies the axioms of probability theory.

The issue in the application of Bayes theory to inference about models and their predictions is how to choose a likelihood function to reflect the evidence contained in a set of model residuals when there are multiple sources of uncertainty in the modelling process. Hierarchical Bayesian methods do so by providing a representation of all of the important sources of uncertainty as aleatory. These representations will then have "hyper-parameters" that are estimated as part of the inference process, based on the series of model residuals. It is critical to this process, however, that all the sources of error can be treated as if they are aleatory.

This is not, however, the case. Many of the errors that enter into the modelling process are not the result of random natural variation but the result of a lack of knowledge about processes and boundary conditions. These epistemic uncertainties are, as pointed out in the 1920s by Frank Knight and Maynard Keynes, the "real" uncertainties. They include the poor measurements, the processes that we have left out because they are not deemed to be important (or for which we have no agreed mathematical representation), and the local catchment characteristics that we cannot know in detail. They also include the "unknown unknowns" that we have not even perceived as being important because of lack of knowledge and which therefore are unexpected (and not predicted) when they occur (these are also sometimes referred to as ontological or irreducible uncertainties). We cannot be too concerned about the latter since past surprises have probably been incorporated into present models and future surprises are difficult to predict. In fact, an ontological uncertainty will become an epistemic uncertainty as soon as it is recognised as an issue. We should, however, be directly concerned about the category of epistemic uncertainties, that we can surely perceive but do not know enough about.

\section{Colour and spin in epistemic errors}

There are very many epistemic errors of this type (see Fig. 1), from spatial patterns of rainfall inputs that vary from storm to storm, to the water equivalent of drifting snow, to radar rainfalls subject to multiple (deterministic) corrections, to rating curve non-stationarities and extrapolations, to evapotranspiration fluxes in hetereogeneous terrain. Some of these may be due to natural variability (as well as the limitations of available measurement techniques to observe that variability) but none can be considered as fundamentally aleatory. Such variability will not be "white" but structured or coloured in a variety of ways and it is a significant spin of our understanding to try to suggest to users of model predictions that they can be treated as if they were aleatory in order to be "objective".

The general effect of epistemic uncertainty is to make the error characteristics of the variable under study (whether input or model residual) to appear structured. If that structure is a well-behaved red noise with stationary characteristics then a statistical model might be found to represent it (e.g. $\mathrm{Li}$ et al., 2011). The issue with epistemic error is, however, that it is likely to induce colour that is non-stationary. A classic example is the way in which an error in an input variable when processed through a nonlinear model, produces a set of residuals that exhibit bias and autocorrelation that might vary depending on the model structure. That is a logical consequence of the nature of the model dynamics. Since the errors in the inputs are expected to vary from event to event (but not in a random way) that bias and autocorrelation will be non-stationary, and normally gradually reducing in effect over time. The colour will change over time. It is known that in simple statistical inference, the neglect of bias and autocorrelation in error series produces bias in the inferred parameters (e.g. Beven et al., 2008). We should therefore expect the same in more complex cases involving non-stationarity.

In extreme cases, the data being used to drive a model or evaluate the predictions might even be adding disinformation to the inference process (Beven and Westerberg, 2011). In hydrological modelling this would be the case when a rainfall input is so poorly represented by a set of raingauges that there is no way that a model could conceivably provide a good estimate of the resulting discharge (see the example in Beven, 2009a). The same could happen if an unverified extrapolation of a discharge rating curve resulted in a 


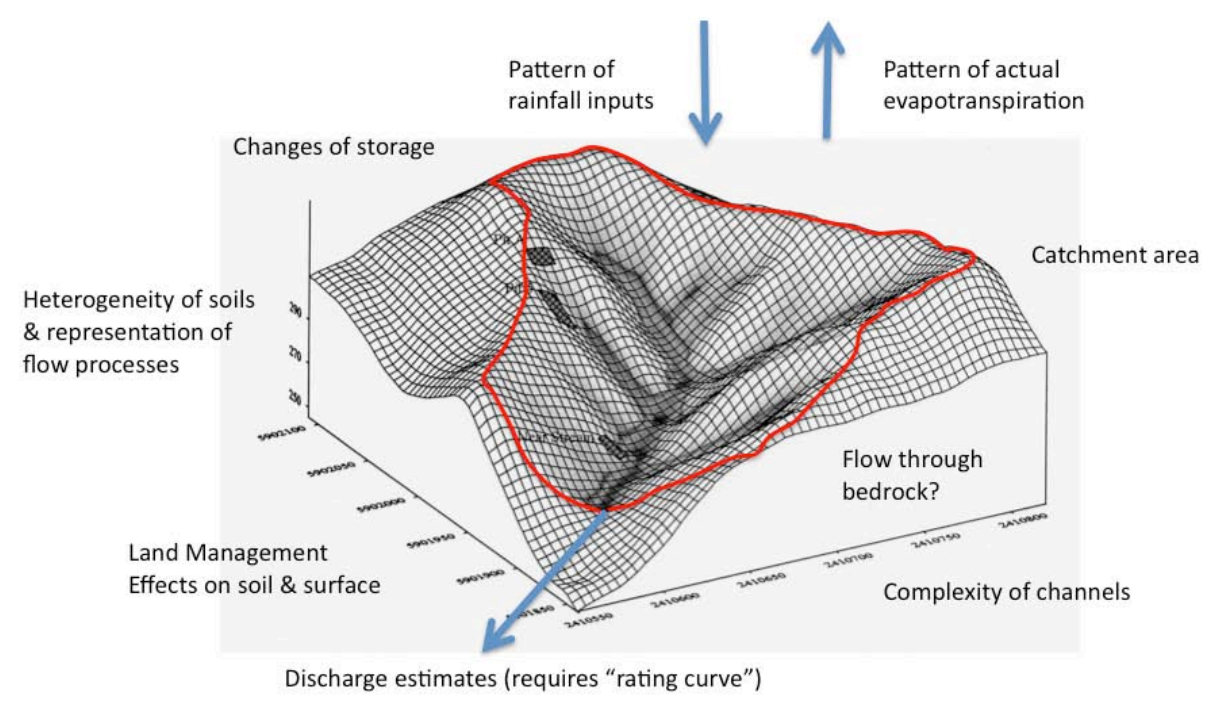

Fig. 1. Potential sources of epistemic error in measuring and modelling catchment responses.

significant overestimation (or underestimation) of discharges at flood stages (modellers should be wary of simply accepting discharge values provided by monitoring Agencies without information about quality control and uncertainties, see Westerberg et al., 2011a). Such errors will not be simply statistical (even where a statistical technique such as regression is used to fit a rating curve to observations). Using any variance-based likelihood function or performance measure that is based on squared residuals (including the NashSutcliffe efficiency measure) might mean that the inference is biased because of just a few periods of such measurements. In rapidly responding catchments timing discrepancies in either the rainfalls or discharges that affect the apparent timing of the rising limb might also have a significant effect on the apparent residual variance. A timing error in the onset of snow melt can have a persistent effect on the error in model simulations that is specific to that period (e.g. see the example in Freer et al., 1996).

Distributed models are particularly interesting in respect of epistemic errors. Distributed models allow for model parameters to vary spatially in every solution element (though this is rarely done, and where it has been done by interpolation it has not generally been very successful, see Loague et al., 2005). Deeper in the subsurface, distributed models can also allow the geological structure to be reflected in the patterns of parameters. There will be epistemic uncertainty in the detail of that structure and it is usually the case that the choice of a "conceptual model" of the geology (and the first estimates of the associated hydrogeological parameters) is a subjective interpretation based on limited amounts of map and geophysical survey data. This is a case where the epistemic uncertainty is treated in terms of one or more possible scenarios (see the case study of Refsgaard et al., 2006).
It is, of course, rare that model parameters can be identified for all the solution elements in the discretisation. It is much more common to fix parameters over some part of the domain. This means that local predictions will be in error but because of the complex nonlinear interactions in space between elements and process representations, the effect of this on model residuals will be difficult to quantify. The effect should certainly not, however, be expected to be aleatory, because of the connected, nonlinear, nature of the distributed simulation model. The colour should not, therefore, be expected to be simple in nature.

\section{Colour, spin and information content}

The reason why this is important is because any colour reduces the information content of the data that are available. Thus, the inference is likely to be over-conditioned if the colour is neglected or represented as if it was a simple stationary process. The result will be generally poorer performance in prediction than in calibration because the characteristics of the sources of uncertainty in prediction will be different to the calibration data. Epistemic errors are expected to lead to this type of non-stationarity. It is indeed generally accepted in hydrological modelling (and in published results of hydrological models) that performance will be poorer in "validation", even after "optimisation" of a model in calibration (though it is probably doubtful if many studies where performance is very much poorer in validation actually get published; it is more likely that a further model iteration takes place).

This expectation of poorer performance in validation is a form of implicit recognition of the effects of epistemic error on the modelling process. In calibration we have no information about how the errors in prediction might be different 
from those in calibration. We can therefore only assume that the characteristics will be in some sense "similar" in prediction. In the case of statistical inference, this is to assume that the parameters of the error models fitted in calibration will be constant in prediction (despite the evidence that they might be difficult to identify in calibration, see Beven, 2005 and Beven et al., 2008). In GLUE it is to assume that the likelihood weights associated with a model parameter set (with its implicit error characteristics) will stay the same in prediction. This does not protect against the unexpected (an example, from the extensively modelled Leaf River data set in the paper by Vrugt et al., 2008, led to the comment of Beven, 2009a).

It is therefore a modelling aphorism that a calibration data set can only be partially informative in the face of epistemic errors. There is no theory of information content to cover such cases. Hence the attraction of spinning the nature of sources of uncertainty to suggest that they can be treated as if they were aleatory. Statistical inference does provide a theory of information content. Classical Gaussian theory, for example, allows individual residual errors to contribute to the likelihood function as:

$L \propto \exp \left(-\rho_{t} e_{t}^{2}\right)$

where $e_{t}$ is the residual error at time $t$. amd $\rho_{t}$ a scaling factor. In the simplest case of an error model that can be assumed to be zero mean and Gaussian with no autocorrelation, this leads to the likelihood function:

$$
\prod_{t} \frac{1}{\sqrt{2 \pi} \sigma} \exp \left(-\frac{1}{2 \sigma^{2}} e_{t}^{2}\right)
$$

where $\sigma^{2}$ is the residual variance. In this framework every residual is considered to be informative (albeit with contributions that can be weighted according to $\rho_{t}$ ). Indeed some hydrological modellers would require this to be the case (e.g. Mantovan and Todini, 2006) for the inference to be "coherent" (see also the response of Beven et al., 2008). The smaller the error, the higher the contribution, but the multiplicative effect over a large number of time steps is to produce a highly peaked likelihood surface, with the danger therefore that where the assumptions of the error model on which the likelihood function is based are not correct (Eq. 2 would not be appropriate for the autocorrelated residuals of most hydrological models, for example), then the contribution to the information content of individual residuals is being overestimated.

As Tarantola (2005) for example points out, the assumption in Eq. (1) is subjective (see also Beven, 2002, 2009b). It is not the only choice about how far a residual contributes information to the conditioning process. Laplace (1774) for example developed an alternative theory based on the absolute values of residuals, i.e.:

$L \propto \exp \left(-\left|\rho_{t} e_{t}\right|\right)$
Since this was mathematically less tractable, it was largely dropped in favour of the Gaussian measure. But neither is a truly objective assessment of information content. They are only objective within the context of the basic assumptions of Eqs. (1) and (3). This applies also to other error norms, or subjective choices of likelihood, that might be chosen.

Such measures should therefore be treated more as hypotheses about future performance to be tested. Statistical error models then provide a formal expectation of performance (in terms of the probability of predicting a future observation) that can be evaluated in terms of actual performance (e.g. in terms of quantile-quantile plots for new prediction periods). Models chosen on the basis of subjective likelihood measures do not have such a probabilistic expectation (as noted earlier, the interpretation of the resulting uncertainty bounds is different). There is an assumption, however, that the characteristics of the errors in prediction should be "similar" to those seen in calibration (see, for example, Liu et al., 2009). Such an assumption can be tested in a similar way. Departures from the range of model predictions (in both calibration and prediction periods) might be useful in identifying consistent model structural error or non-stationary epistemic error (see, for example, Westerberg et al., 2011b).

There is no real reason why a more direct recognition of epistemic errors should not be based on choices about information content in a way that allows for the expectation of future variability in error characteristics. The difficulty in doing so is that epistemic errors are those for which, by definition, we have little or no information about their nature in calibration and even less about the potential errors in prediction.

What we can do, however, is list some desirable qualities or principles for an assessment of information content when we suspect that the modelling process is subject to structural error. These should then be reflected in any model evaluation and likelihood assessment. In their strongest form these principles might be expressed as:

1. Information should be assessed so as to minimise, as far as possible, Type I (false positive, or accepting a model that would not provide useful predictions) and Type II (false negative, or rejecting a model that would provide useful prediction) errors.

2. Periods of disinformative inputs or outputs should be identified as far as possible independently of any model structure to avoid the reductio ad absurdum of all periods that do not fit a particular model being rejected as disinformative.

3. Contributions to model likelihood evaluations should be a function of the time varying information content of the observations.

4. Account should be taken of the potential for input errors to have an effect on model outputs over multiple consequent events. 
These features may be desirable but will be difficult to satisfy because of the expectation that the epistemic errors will be non-stationary and of complex structure, while principle 2 precludes the use of model residuals in assessing information content in this sense. It is difficult therefore to define a strategy for the independent estimation of such errors without additional information being provided (which generally is not possible for periods of past calibration data).

\section{Avoiding false negatives}

The first principle above is important. It is a fundamental principle for the assessment of information content. It is worth noting, however, that false negative errors are much more important than false positives. The potential for false positives is the reason underlying principle 2 , but false positives will generally be less serious because they can be corrected as more information becomes available. However, once a model that would provide useful predictions in the future is rejected (a false negative), those useful predictions will not then be available. There is little experience in testing hydrological models as hypotheses within such a framework (Beven, 2010).

False positive and false negative inferences can be expected to result when the driving data for a model are hydrologically inconsistent with the observed variables with which model simulated variables will be compared. We might expect such an inconsistency to be expressed in terms of large model residuals (even for a model that might be useful in prediction) but, following principle 2 , we would ideally wish to assess consistency independently of the model hypothesis being evaluated.

So what does hydrological consistency mean? That the inputs and outputs should be consistent with the mass, energy and momentum balances and what is known about the processes in a catchment. But these are difficult to assess. As part of the Representative Elementary Watershed concepts, Reggiani and Schellekens (2003, see also Reggiani et al., 2000,2001 ) have shown that the mass, energy and momentum balances can be expressed for catchment or subcatchment units but that they are subject to an important closure problem. Expressed in terms of the simplest lumped catchment water balance for example, we can classically write:

$Q=R+E+\Delta S$

where $Q$ is discharge, $P$ is all types of precipitation, $E$ is evapotranspiration rate and $\Delta S$ is the integral change in storage for all points and at all levels in the catchment. In fact, even this is too simplistic, since there may be unmeasured discharges, $U$, from a catchment (due to regional groundwater fluxes, or unmeasured subsurface fluxes beneath a gauging site). Thus:

$Q=R+E+\Delta S+U$
In addition each of these terms would be subject to estimation errors, particularly over short periods of time (such as those for which we might wish to assess the information content of data). Allowing for such uncertainties in observed values of the variables is, in general and even for experimental catchments, the only way in which the water balance can be closed. In practice, therefore, inconsistencies can only be assessed in the broadest sense of departures from the behaviour seen in the data series as a whole.

In keeping with principle 2 therefore, is there a way of identifying periods of inconsistent data independent of any model structure being evaluated? Two strategies (at least) would seem feasible. One is to use a non-parametric method for explaining relationships in the observations themselves, such as the regression tree methodology used by Iorgulescu and Beven (2004). Past experience suggests that this can be useful in identifying anomalous periods of data. The second would be to identify a characteristic response function for a catchment (the unit hydrograph) and test for anomalies in runoff coefficients for individual storms (allowing for the antecedent state of the catchment and seasonal effects in the form of accumulated evapotranspiration since the previous event).

There are dangers in both of these approaches, in that "unusual" periods in the observations, if they were in fact hydrologically consistent, would probably be the most informative in differentiating between model hypotheses. There is therefore a need to differentiate between hydrologically consistent and hydrologically inconsistent anomalies (with analogous possibilities of being wrong as a result of uncertainty). Remember, however, that we should expect not to be sure about identifying hydrological inconsistencies in all cases. There will always be the potential for making Type I and Type II errors.

More generally, principle 3 suggests that it might be possible to rank observations or periods of observations in terms of their contribution to total information content in conditioning a model. This implies a more continuous scale of information than simply excluding certain periods of data considered to be disinformative. Such a ranking, or weighting, of different periods of data might be expected to be context dependent, with relevance to the particular type of application of interest and to the expected observation errors (the classical statistical weighting as an inverse function of observation variance is an example of this). It is also the case that the same set of observations might have more information content with respect to one model output or component than to another. There has been some (limited) exploration of this concept already in hydrological modelling, with Gupta et al. (2008); Wagener et al. (2003).

Principle 4 is important. Model output uncertainties in hydrology and hydraulics are often dominated by input data uncertainties, due to limited measurement sites in space or time or both. Thus the information content of accurate output observations that are being used to evaluate model performance 
might be compromised by input uncertainties that, in many cases, are very difficult to characterise because of heterogeneities and non-stationarities between events. It was this type of uncertainty that was the issue at the heart of the discussion between Beven (2009a) and Vrugt et al. $(2008,2009)$ and that was illustrated by Beven and Westerberg (2011).

\section{An example}

The above points can be illustrated by considering an example, the calibration of a rainfall-runoff model to observed data. The observed data are those readily available in the UK, discharges from an Environment Agency stream gauge and hourly rainfall totals from Met Office rain gauges. The catchment is the South Tyne station 23006 at Featherstone $\left(322 \mathrm{~km}^{2}\right)$. This catchment is predominantly moorland vegetation and the land-use is mainly for rough grazing. The geology is mainly Carboniferous limestone. An important consideration for the choice of this catchment was that the flows for this site are natural to within $10 \%$ at the 95 percentile flow. Five recording rain gauges are available within the catchment boundary of the chosen site, with catchment average rainfalls being estimated using a Thiessen polygon method. Comparison with storage gauges in the catchment revealed that the recording gauges generally estimated higher rainfall totals than the storage gauges. There is scope for both the under and overestimation of the inputs to the catchment under different rainfall patterns.

Discharges are measured using a compound Crump weir, which contains the flow at all stages and remains modular throughout its range. There is some suspicion of truncation of higher peaks, possibly due to stilling well problems, but the discharge data are generally considered to be of good quality and are included in the UK HiFlows database.

Periods of disinformative data were assessed though the use of event runoff coefficients. Variability in event runoff coefficients is to be expected due to inadequacies in observing both the precipitation input and the output discharge as well as differing internal states of the system. However, events whose runoff coefficients differ substantially from similar events (e.g. those with similar rainfall totals falling in the same season) should be further investigated. In some cases these may be examples of catchment dynamics not often observed so should remain in the analysis; in other cases they may be disinformative.

\subsection{Selection of disinformative periods}

To perform the selection of disinformative data periods on an event basis, the observed data needs to be broken into events for which the total runoff and precipitation can be computed. Calculating the total runoff requires the extrapolation of the falling limb of the event hydrograph to estimate the volume

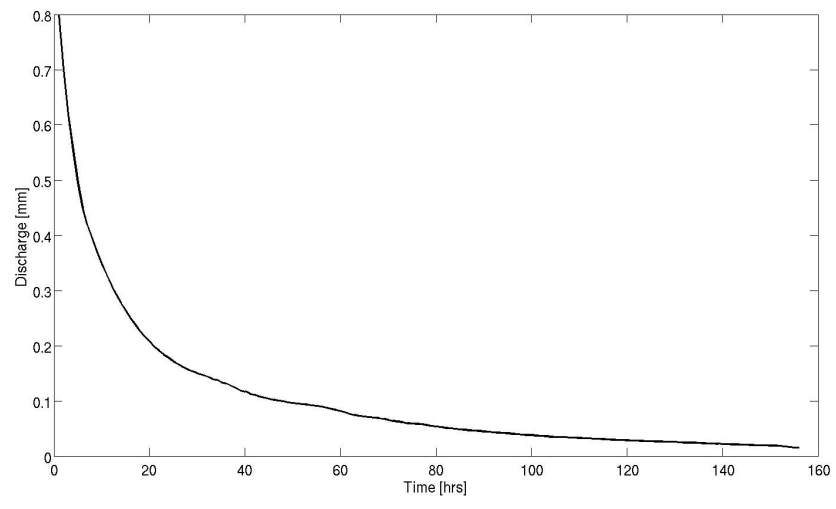

Fig. 2. Plot of the lower part of the master recession curve.

of discharge that might have been observed if further rainfall had not occurred.

The analysis of recession curves has a long history in hydrology and extensive reviews have been provided by Hall (1968) and Tallaksen (1995). In spite of well-known difficulties with recession variability and partitioning of flow sources, an analysis of recession curves can often give some indication of the characteristics of the subsurface discharges from a catchment and can be used to develop catchment storage models (Lamb and Beven, 1997).

The approach taken for this study was to develop a master recession curve (MRC) by piecing together individual shorter recession curves. Ideally, only true flow recessions should be selected where there is no rainfall and minimal evapotranspiration during the flow recession period. In practise this ideal is difficult to achieve and in this study the MRC was constructed by piecing together individual recession curves of greater than $12 \mathrm{~h}$ duration during which less then $0.2 \mathrm{~mm}$ of rainfall fell. Figure 2 shows the lower part of the MRC.

Figure 3 outlines the calculation of the runoff volume for an event. The time period was divided into events. An event was considered to start at the first rainfall after 12 dry hours (hours with rainfall less then $0.2 \mathrm{~mm}$ ) when the discharge at that time was less then $0.8 \mathrm{~mm}$ and so within the range of a parametric approximation to the MRC. Figure 4 shows the runoff coefficient for the 1817 events plotted against the total event rainfall. From this it is clear that the runoff coefficient for small events is often poorly estimated. While the approximation of the MRC at low discharges may play a role in this, the misobservation of low catchment rainfall totals, which may indicate localised patterns of rainfall over the catchment, is felt to be the dominant cause. These events are deemed disinformative. A number of rainfall events with larger totals (Fig. 4) also show unreasonable runoff coefficients (greater than 0.95). For whatever reason, these events are hydrologically inconsistent and are also deemed disinformative as are those with a runoff coefficient of less than 0.05 in this upland UK catchment. These limits on the runoff coefficients are such that some inconsistent events might still be 
Table 1. Table showing the number of Monte Carlo samples considered behavioural for varying thresholds. Values for the data series with disinformative periods removed are shown in brackets.

\begin{tabular}{lrrrrrr}
\hline$\kappa_{\min }$ & \multicolumn{5}{c}{$\kappa_{\max }$} \\
\cline { 2 - 7 } & 1 & 0.99 & 0.98 & 0.97 & 0.95 & 0.93 \\
\hline-100 & $10152(10192)$ & $5819(9544)$ & $456(6082)$ & $145(2968)$ & $22(1408)$ & $1(789)$ \\
-50 & $8733(8754)$ & $4885(8201)$ & $333(5213)$ & $111(2561)$ & $16(1175)$ & $1(641)$ \\
-30 & $7459(7478)$ & $4095(7011)$ & $235(4442)$ & $81(2181)$ & $12(977)$ & $1(507)$ \\
-10 & $4192(4214)$ & $2210(3920)$ & $86(2471)$ & $25(1262)$ & $2(496)$ & $0(215)$ \\
-5 & $2276(2308)$ & $980(2090)$ & $13(1162)$ & $6(549)$ & $1(164)$ & $0(56)$ \\
\hline
\end{tabular}

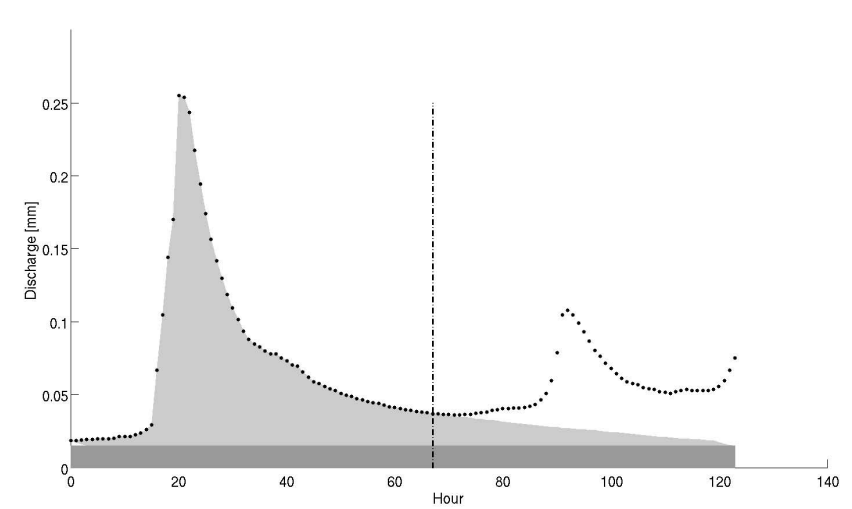

Fig. 3. Schematic showing the calculation of the runoff volume associated with an event. The lighter shaded area represents the runoff volume associated with the event which starts at time 0 and the darker shaded area an underlying baseflow The subsequent events starts after $67 \mathrm{~h}$ (solid line) so the lighter shaded area after this time is extrapolated using the MRC.

accepted (the mean runoff coefficient over the whole period in this catchment is 0.8 ).

\subsection{Influence of disinformative data on calibration}

Consider calibrating a lumped hydrological model to the observed flow series. The effects of the disinformative data on the calibration of a hydrological model can be illustrated using the lumped rainfall runoff model outlined in Eqs. (4) and (5). The model is Hammerstein in form representing a non-linear transform of the input rainfall series (Eq. 4) and then routing through two parallel tanks expressed as a linear transfer function. The linear transfer function is assumed to be mass conservative so that the parameterisation $\left(\alpha_{1}, \alpha_{2}, \beta_{1}, \beta_{2}\right)$ can be simplified to $\left(\alpha_{1}, \alpha_{2}, \rho\right)$ where $\rho$ is the split fraction of effective input entering the first path. To ensure the model is physically meaningful $\left(\alpha_{1}, \alpha_{2}, \rho, \phi_{1}\right)$ lie between 0 and 1 , while $\phi_{2}$ is greater than 0 (the range 0 to 2 is considered). To ensure identifiability the condition $\alpha_{2}>\alpha_{1}$ is imposed.

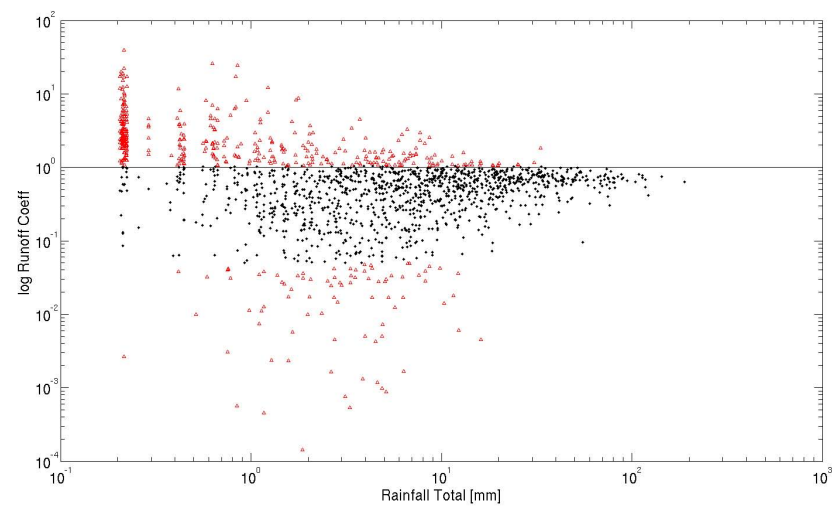

Fig. 4. Scatter plot of runoff coefficient against total rainfall for the 1817 events identified in the time period. Those in red are considered as disinformative.

$u_{t}=\phi_{2} y_{t}^{\phi_{1}} r_{t}$

$x_{t}=\frac{\beta_{1}}{1-\alpha_{1}} u_{t-2}+\frac{\beta_{2}}{1-\alpha_{2}} u_{t-2}$

Twelve thousand uniformly distributed random samples were drawn from the parameter ranges outlined above. For each parameter set the maximum and minimum values of the scaled residual $\left(y_{t}-x_{t}\right) / y_{t}$ were computed for: (a) the first 900 events identified in the time series and (b) those events in the first 900 that are not excluded as disinformative. Parameter sets were considered behavioural if

$\min _{t} \frac{y_{t}-x_{t}}{y_{t}}>\kappa_{\min }$

and

$\max _{t} \frac{y_{t}-x_{t}}{y_{t}}<\kappa_{\max }$.

Table 1 shows the number of parameter sets considered behavioural for various combinations of $\kappa_{\min }$ and $\kappa_{\max }$. These indicate that by including the disinformative data in the analysis there may be a risk of making a Type II error for given values of $\kappa_{\min }$ and $\kappa_{\max }$. Moreover the results show 


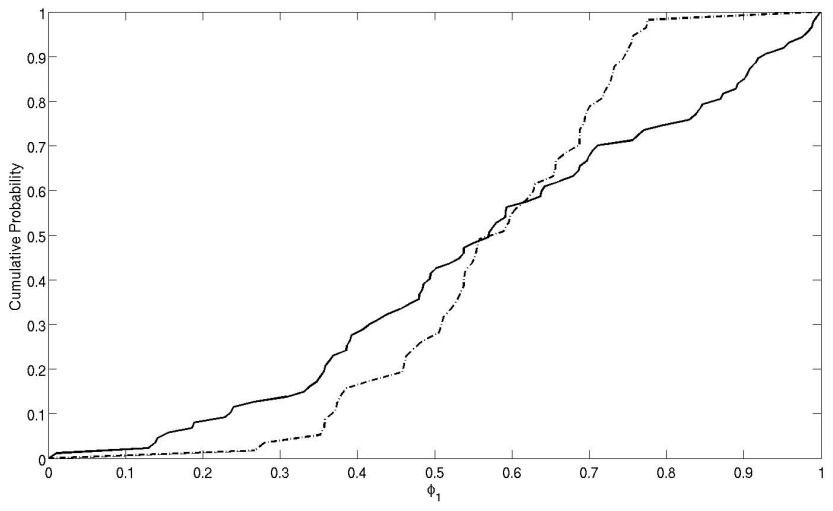

Fig. 5. Cumulative distribution of $\phi_{1}$ for behavioural parameter sets of the two situations considered: Analysis using the full data set with $\left(\kappa_{\min }, \kappa_{\max }\right)=(-10,0.98)$ (solid) and with the disinformative data removed with $\left(\kappa_{\min }, \kappa_{\max }\right)=(-5,0.93)($ dot-dash $)$.

that the model which is not considered behavioural for say $\left(\kappa_{\min }, \kappa_{\max }\right)=(-10,0.98)$ may be considered behavioural for stricter conditions (e.g. $\left(\kappa_{\min }, \kappa_{\max }\right)=(-10,0.9)$ when the disinformative data is removed.

Specification of the behavioural threshold can therefore affect the potential for making Type II errors, but specifying too generous a bound will also increase the possibility of Type I errors. To illustrate how these trade-offs may influence the conclusions drawn from analysis of the model two situations are contrasted. Situation 1 relates to considering the full calibration data set and specifying behavioural limits of $\left(\kappa_{\min }, \kappa_{\max }\right)=(-10,0.98)$. The second situation considers analysis using only the informative data and behavioural thresholds of $\left(\kappa_{\min }, \kappa_{\max }\right)=(-5,0.93)$.

Figures 5 and 6 show that the resulting distribution of the behavioural parameters sets may change both in location and spread. Figure 7 shows how the selection of the threshold may influence the output of the behavioural model simulations for several events outside the calibration period. The simulation results indicate that the very simple model used is structurally deficient, failing to capture the changes in timing in the rising limb of the hydrograph while tending to overestimate the peak discharge.

\section{Back to the unexpected future}

In calibration, therefore, we can attempt to identify periods of data that might be disinformative in model inference independent of model runs. We should not expect a behavioural model to predict such periods of data (while recognising that we might still be making Type I errors in accepting some models as behavioural). There might be similar periods of hydrologically inconsistent data in prediction, that can be identified in similar ways to those applied in calibration. It therefore follows that we should not expect such periods to

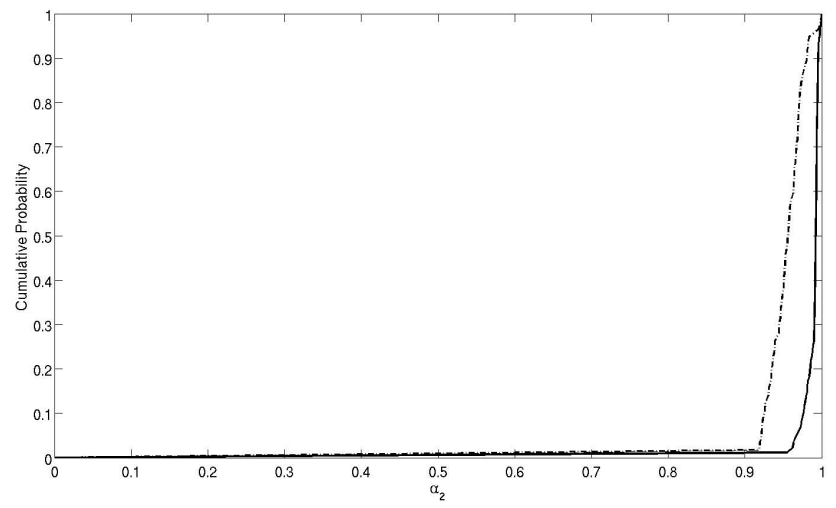

Fig. 6. Cumulative distribution of $\alpha_{2}$ for behavioural parameter sets of the two situations considered: Analysis using the full data set with $\left(\kappa_{\min }, \kappa_{\max }\right)=(-10,0.98)$ (solid) and with the disinformative data removed with $\left(\kappa_{\min }, \kappa_{\max }\right)=(-5,0.93)($ dot-dash $)$.

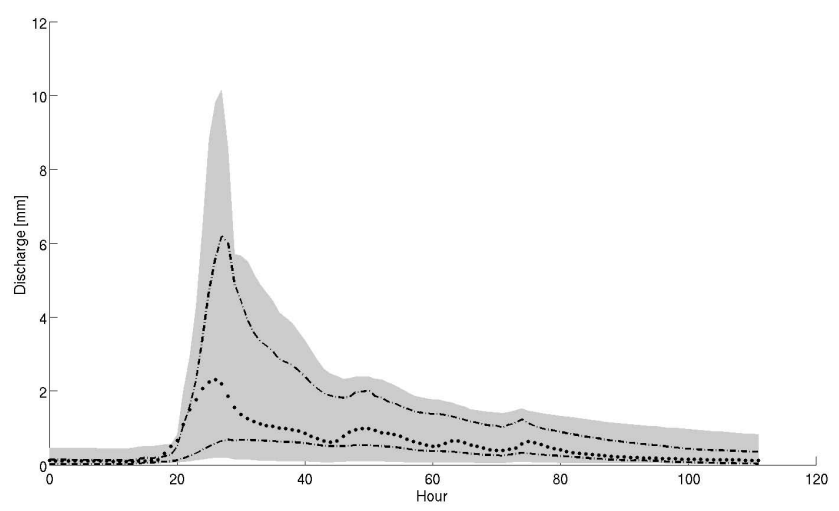

Fig. 7. Simulation results for an event not within the calibration period. the shaded area represents the limits (evaluated on a time step by time step basis) of the simulations deemed to be behavioural when calibrated against all the calibration data with $\left(\kappa_{\min }, \kappa_{\max }\right)=(-10,0.98)$. The lines correspond to the limits when on the informative calibration data is used and $\left(\kappa_{\min }, \kappa_{\max }\right)=(-5,0.93)$.Points represent the observed data.

be well predicted by the set of behavioural models identified in calibration. We should also not expect that such periods would be covered by any statistical representation of the calibration errors, since the epistemic uncertainties of inconsistent periods in prediction might be quite different to those in calibration. The only response to this would appear to be to moderate our expectations of what a model, or set of models, can do in prediction.

Once new observations are available, and they have been checked for consistency, they can be used in further testing of the set of behavioural models. As noted earlier, new observations might reduce the potential for Type I errors by rejecting some of the set of behavioural models based on the new evidence. New observations then contribute to the type of learning process suggested in Beven et al. (2007) and Buytaert and Beven (2009). 
We might expect the uncertainty in the driving variables for hydrological and hydraulic models to be reduced by the application of new or more pervasive measurement techniques into the future. The next generation of radar estimates of rainfalls or satellite estimates of surface soil moisture will, we are assured, provide greater accuracy and finer resolution. It might still be expected, however, that some significant epistemic uncertainties in model inputs and model structures will remain for the foreseeable future.

We think we have a pretty good perceptual model of how catchment systems work such that many of the epistemic uncertainties discussed above arise, not from unperceived unknowns, but from the limitations of current measurement techniques, spatial and temporal sampling, estimating effective parameter values etc. But, of course, this is true for every generation, and then some unexpected information comes along to change that impression. For the early computer modelling generation, one of the most important unexpected pieces of information was the introduction of tracer data that allowed the residence times in catchments to be addressed in ways not previously possible (e.g. Sklash and Farvolden, 1979). Suddenly, subsurface flows could not be ignored (see also the history of the R5 modelling exercise reported in Loague and Vanderkwaak, 2002 and modelling the Plynlimon chloride data in Kirchner et al., 2001 and Page et al., 2007).

What we can be sure of is that the next generation of hydrological modellers will also have access to new and better geophysical and geochemical information, and that their perceptions of how hydrological systems work will change. There remain some epistemological uncertainties of which, as yet, we have only the vaguest intimation but which we should expect to be reduced in achieving better hydrological simulations and improved integrated catchment management in the future.

\section{Conclusions}

Disinformation as a result of epistemic error is an issue in hydrological modelling. In particular the way in which the colour in model residuals resulting from epistemic errors should be expected to be non-stationary means that it is difficult to justify the spin that the structure of residuals can be properly represented by statistical likelihood functions. To do so would be to greatly overestimate the information content in a set of calibration data and increase the possibility of both Type I and Type II errors. This has been recognised in the past in the bias to be expected in posterior parameter distributions when too simplistic a likelihood function is used, but it has been suggested here that the problem is much more significant. Some techniques have been suggested for identifying periods of disinformative data prior to evaluation of a model structure of interest, and the effect on the estimated parameter values of a hydrological model has been demonstrated.
Acknowledgements. This work has been funded by the EPSRC Flood Risk Management Research Consortium project and the EU IMPRINTS FP7 project. We thank both Alberto Montanari and Andras Bardossy for their thoughtful and valuable referee comments and Ida Westerberg for her perceptive suggestions.

Edited by: T. Moramarco

\section{References}

Andréassian, V., Lerat, J., Loumagne, C., Mathevet, T., Michel, C., Oudin, L., and Perrin, C.: What is really undermining hydrologic science today?, Hydrol. Process., 21, 2819-2822, 2007.

Bayes, T.: An essay towards solving a problem in the doctrine of chances, Philos. T. Roy. Soc. Lond., 53, 370-418, 1763.

Beven, K. J.: Towards a coherent philosophy for modelling the environment, P. Roy. Soc. Lond. A, 458, 2465-2484, 2002.

Beven, K. J.: Robert E. Horton's perceptual model of infiltration processes, Hydrol. Process., 18, 3447-3460, 2004.

Beven, K. J.: On the concept of model structural error, Water Sci. Technol., 52, 165-175, 2005.

Beven, K. J.: A manifesto for the equifinality thesis, J. Hydrol., 320, 18-36, 2006.

Beven, K. J.: Comment on "Equifinality of formal (DREAM) and informal (GLUE) Bayesian approaches in hydrologic modeling?" by Jasper A. Vrugt, Cajo J. F. ter Braak, Hoshin V. Gupta and Bruce A. Robinson, Stoch. Environ. Res. Risk Assess., 23, 1059-1060, 2009a.

Beven, K. J.: Environmental Modelling: An Uncertain Future?, Routledge, London, UK, 2009b.

Beven, K. J.: Preferential flows and travel time distributions: defining adequate hypothesis tests for hydrological process models Preface, Hydrol. Process., 24, 1537-1547, 2010.

Beven, K. J. and Binley, A. M.: The Future of Distributed Models Model Calibration and Uncertainty Prediction, Hydrol. Process., 6, 279-298, 1992.

Beven, K. J. and Westerberg, I.: On red herrings and real herrings: disinformation and information in hydrological inference, Hydrol. Process., 25, 1676-1680, doi:10.1002/hyp.7963, 2011.

Beven, K. J., Smith, P. J., and Freer, J.: Comment on "Hydrological forecasting uncertainty assessment: incoherence of the GLUE methodology" by Pietro Mantovan and Ezio Todini, J. Hydrol., 338, 315-318, 2007.

Beven, K. J., Smith, P. J., and Freer, J.: So just why would a modeller choose to be incoherent?, J. Hydrol., 354, 15-32, 2008.

Buytaert, W. and Beven, K. J.: Regionalization as a learning process, Water Resour. Res., 45, W11419, doi:10.1029/2008WR007359, 2009.

Freer, J., Beven, K. J., and Ambroise, B.: Bayesian estimation of uncertainty in runoff prediction and the value of data: An application of the GLUE approach, Water Resour. Res., 32, 21612173, 1996.

Goldstein, M. and Rougier, J.: Probabilistic formulations for transferring inferences from mathematical models to physical systems, Siam J. Sci. Comput., 26, 467-487, 2004.

Gupta, H. V., Wagener, T., and Liu, Y. Q.: Reconciling theory with observations: elements of a diagnostic approach to model evaluation, Hydrol. Process., 22, 3802-3813, 2008. 
Hall, F. R.: Base-flow Recessions-a Review, Water Resour. Res., 4, 973-983, doi:10.1029/WR004i005p00973, 1968.

Hornberger, G. M. and Spear, R. C.: An Approach To the Preliminary-analysis of Environmental Systems, J. Environ. Manage., 12, 7-18, 1981.

Iorgulescu, I. and Beven, K. J.: Nonparametric direct mapping of rainfall-runoff relationships: An alternative approach to data analysis and modeling?, Water Resour. Res., 40, W08403, doi:10.1029/2004WR003094, 2004.

Kennedy, M. C. and O'Hagan, A.: Bayesian calibration of computer models, J. Roy. Stat. Soc. B, 63, 425-450, 2001.

Kirchner, J. W., Feng, X. H., and Neal, C.: Catchment-scale advection and dispersion as a mechanism for fractal scaling in stream tracer concentrations, J. Hydrol., 254, 82-101, 2001.

Kuczera, G., Kavetski, D., Franks, S., and Thyer, M.: Towards a Bayesian total error analysis of conceptual rainfall-runoff models: Characterising model error using storm-dependent parameters, J. Hydrol., 331, 161-177, 2006.

Lamb, R. and Beven, K.: Using interactive recession curve analysis to specify a general catchment storage model, Hydrol. Earth Syst. Sci., 1, 101-113, doi:10.5194/hess-1-101-1997, 1997.

Laplace, P.: Mémoire sur la probabilité des causes par les évènements, Mémoires de l'Academie de Science de Paris, 6, 621-656, 1774.

Li, L., Xu, C.-Y., Xia, J., Engeland, K., and Reggiani, P.: Uncertainty estimates by Bayesian method with likelihood of AR (1) plus Normal model and AR (1) plus Multi-Normal model in different time-scales hydrological models, J. Hydrol., 406, 54-65, doi:10.1016/j.jhydrol.2011.05.052, 2011.

Liu, Y., Freer, J. E., Beven, K. J., and Matgen, P.: Towards a limits of acceptability approach to the calibration of hydrological models: extending observation error, J. Hydrol., 367, 93-103, doi:10.1016/j.jhydrol.2009.01.016, 2009.

Loague, K. and Vanderkwaak, J. E.: Simulating hydrological response for the R-5 catchment: comparison of two models and the impact of the roads, Hydrol. Process, 16, 1015-1032, 2002.

Loague, K., Heppner, C. S., Abrams, R. H., Carr, A. E., VanderKwaak, J. E., and Ebel, B. A.: Further testing of the Integrated Hydrology Model (InHM): event-based simulations for a small rangeland catchment located near Chickasha, Oklahoma, Hydrol. Process., 19, 1373-1398, 2005.

Mantovan, P. and Todini, E.: Hydrological forecasting uncertainty assessment: Incoherence of the GLUE methodology, J. Hydrol., 330, 368-381, 2006.

O'Hagan, A. and Oakley, J. E.: Probability is perfect, but we can't elicit it perfectly, Reliabil. Eng. Syst. Saf., 85, 239-248, 2004.

Page, T., Beven, K. J., Freer, J., and Neal, C.: Modelling the chloride signal at Plynlimon, Wales, using a modified dynamic TOPMODEL incorporating conservative chemical mixing (with uncertainty), Hydrol. Process., 21, 292-307, 2007.

Refsgaard, J. C., van der Sluijs, J. P., Brown, J., and van der Keur, P.: A framework for dealing with uncertainty due to model structure error, Adv. Water Res., 29, 1586-1597, 2006.

Reggiani, P. and Schellekens, J.: Modelling of hydrological responses: the representative elementary watershed approach as an alternative blueprint for watershed modelling, Hydrol. Process., 17, 3785-3789, 2003.
Reggiani, P., Sivapalan, M., and Hassanizadeh, S. M.: Conservation equations governing hillslope responses: Exploring the physical basis of water balance, Water Resour. Res., 36, 1845-1863, 2000.

Reggiani, P., Sivapalan, M., Hassanizadeh, S. M., and Gray, W. G.: Coupled equations for mass and momentum balance in a stream network: theoretical derivation and computational experiments, P. Roy. Soc. Lond. A, 457, 157-189, 2001.

Renard, B., Kavetski, D., Kuczera, G., Thyer, M., and Franks, S. W.: Understanding predictive uncertainty in hydrologic modeling: The challenge of identifying input and structural errors, Water Resour. Res., 46, W05521, doi:10.1029/2009WR008328, 2010.

Sklash, M. G. and Farvolden, R. N.: Role Of Groundwater In Storm Runoff, J. Hydrol., 43, 45-65, 1979.

Smith, P., Beven, K. J., and Tawn, J. A.: Informal likelihood measures in model assessment: Theoretic development and investigation, Adv. Water Resour., 31, 1087-1100, 2008.

Sorooshian, S. and Dracup, J. A.: Stochastic Parameter-Estimation Procedures For Hydrologic Rainfall-Runoff Models - Correlated And Heteroscedastic Error Cases, Water Resour. Res., 16, 430442, 1980.

Stedinger, J. R., Vogel, R. M., Lee, S. U., and Batchelder, R.: Appraisal of the generalized likelihood uncertainty estimation (GLUE) method, Water Resour. Res., 44, W00B06, doi:10.1029/2008WR006822, 2008.

Tallaksen, L. M.: A Review of Baseflow Recession Analysis, J. Hydrol., 165, 349-370, 1995.

Tarantola, A.: Inverse problem theory and model parameter estimation, SIAM, Philadelphia, PA, 2005.

Thyer, M., Renard, B., Kavetski, D., Kuczera, G., Franks, S. W., and Srikanthan, S.: Critical evaluation of parameter consistency and predictive uncertainty in hydrological modeling: A case study using Bayesian total error analysis, Water Resour. Res., 45, W00B14, doi:10.1029/2008WR006825, 2009.

Vrugt, J. A., ter Braak, C., Gupta, H. V., and Robinson, B.: Equifinality of formal (DREAM) and informal (GLUE) Bayesian approaches in hydrologic modeling?, Stoch. Environ. Res. Risk Assess., 23, 1011-1026, doi:10.1007/s00477-008-0274-y, 2008.

Vrugt, J. A., ter Braak, C. J. F., Gupta, H. V., and Robinson, B. A.: Response to comment by Keith Beven on "Equifinality of formal (DREAM) and informal (GLUE) Bayesian approaches in hydrologic modeling?", Stoch. Environ. Res. Risk Assess., 23, 1011-1026, doi:10.1007/s00477-008-0274-y, 2009.

Wagener, T., McIntyre, N., Lees, M. J., Wheater, H. S., and Gupta, H. V.: Towards reduced uncertainty in conceptual rainfall-runoff modelling: Dynamic identifiability analysis, Hydrol. Process., 17, 455-476, 2003.

Westerberg, I., Guerrero, J. L., Seibert, J., Beven, K. J., and Halldin, S.: Stage-discharge uncertainty derived with a non-stationary rating curve in the Choluteca River, Honduras, Hydrol. Process., 25, 603-613, 2011a.

Westerberg, I. K., Guerrero, J.-L., Younger, P. M., Beven, K. J., Seibert, J., Halldin, S., Freer, J. E., and Xu, C.-Y.: Calibration of hydrological models using flow-duration curves, Hydrol. Earth Syst. Sci., 15, 2205-2227, doi:10.5194/hess-15-22052011, $2011 b$. 